\title{
Convergence behaviour of deflated GMRES(m) algorithms on AP3000
}

\author{
Kentaro Moriya Takashi Nodera*
}

(Received 7 August 2000)

\begin{abstract}
GMRES $(\mathrm{m})$ method, the restarted version of the GMRES (generalized minimal residual) method, is one of the major iterative methods for numerically solving large and sparse nonsymmetric problems of the form $A x=b$. However, the information of some eigenvectors that compose the approximation disappears and then the good approximate solution cannot be obtained, because of this restart. Recently, in order to improve such a weak point, some algorithms which
\end{abstract}

*Keio University, 3-14-1 Hiyoshi Kohoku, Yokohama 223-8522, JAPAN

${ }^{0}$ See http://anziamj . austms . org. au/V42/CTAC99/Mori for this article and ancillary services, (C) Austral. Mathematical Soc. 2000. Published 27 Nov 2000. 
named MORGAN, DEFLATION and DEFLATED-GMRES algorithm, have been proposed $[7,10,12]$. Those algorithms add the information of eigenvectors that can be obtained in the previous restart frequency. In this paper, we study those algorithms and compare their performances. From the numerical experiments on the distributed memory machine Fujitsu AP3000, we show that DEFlated-GMREs $(m, k)$ method performs the good reduction of residual norms in these algorithms.

\section{Contents}

1 Introduction

C998

2 Deflated GMRES $(m)$ Algorithms

C999

3 Numerical Experiments

C1001

3.1 Example 1 $\mathrm{C} 1002$

3.2 Example 2

$\mathrm{C} 1006$

3.3 Example 3

4 Concluding Remark

C1013

References

C1016 


\section{Introduction}

We now consider the numerical solution of large and sparse linear systems of equations

$$
A x=b
$$

by using of iterative techniques. Numerical formulations of partial differential equation problems give rise to systems of large sparse nonsymmetric linear systems. For these systems robust and fast iterative algorithms must be used. Now, there are quite a few Krylov subspace algorithms for solving the above linear systems, e.g. GMRES, BiCGStab $(\ell)$ and QMR. Of these algorithms, one kind of the most profitable technique is based on the orthogonal projection, typified by GMRES algorithm.

The GMRES begins with an initial guess $x_{0}$ and characterizes the $k$ th iterate as

$$
x_{k}=x_{0}+y_{k}
$$

where $y_{k}$ solves the least squares problem such as

$$
\min _{y \in \mathcal{K}_{k}\left(r_{0}\right)}\left\|b-A\left(x_{0}+y\right)\right\|_{2}=\min _{y \in \mathcal{K}_{k}\left(r_{0}\right)}\left\|r_{0}-A y\right\|
$$

In equation (2), the initial residual vector $r_{0}=b-A x_{0}$ and for $v \in \mathcal{R}^{n}$, $\mathcal{K}_{k}(v)$ is the Krylov subspace

$$
\mathcal{K}_{k}(v) \equiv \operatorname{span}\left\{v, A v, A^{2} v, \ldots, A^{k-1} v\right\} .
$$


At the centre of the usual implementations of GMRES algorithm is Arnordi process, which is given in Saad et al. [2], to construct an orthonormal basis for the Krylov subspace. For starting the GMRES step, the process is applied with $v_{1}=r_{0} /\left\|r_{0}\right\|_{2}$. At each step, the orthonormalization can be done using the modified Gram-Schmidt process. Each $\left\{v_{1}, v_{2}, \ldots, v_{n}\right\}$ is an orthonormal basis of $\mathcal{K}_{k}\left(r_{0}\right)$ that reduces the least squares problem (2) to an upper Hessenberg least squares problem. This is normally solved by QR decomposition with Givens rotations. So full orthogonalization is needed, but more expensive as the subspace are increased. Moreover, the storage requirements also increase. For such reason, we can usually use restarting when the subspace reaches a certain size of $m$. We call this algorithm the restarted $\operatorname{GMRES}(m)$. The disadvantage with restarting is that some information of eigenvector is lost at the time of the restart. The subspace is cast off, and then restarted GMRES algorithms slow down the convergence of residual norm.

In Section 2, we will briefly recall some related properties of deflated $\operatorname{GmRes}(m)$ algorithms. Next, in Section 3, we illustrate on some results of numerical experiments on AP3000. At last, we give some concluding remarks.

\section{Deflated GMRES $(m)$ Algorithms}

In deflation algorithms, there are two distinct ways of developing some knowledge about approximate eigenvectors of $A M^{-1}$ to accelerate convergence. 
The first approach is just to add the desired eigenvectors directly to the Krylov subspace. For eigenvalue problems, this is absolutely natural [1]. For solving the linear system (1), this approach has been proposed by Morgan [7]. Morgan injects eigenvectors $u_{1}, u_{2}, \ldots, u_{p}$ into Krylov subspace $\mathcal{K}_{k}\left(r_{0}\right)$, so the solution $x$ then belong to

$$
x_{0}+\operatorname{span}\left\{r_{0}, A r_{0}, \ldots, A^{k-p+1} r_{0}, u_{1}, u_{2}, \ldots, u_{p}\right\}
$$

The proposed algorithm is called $\operatorname{MORGAM}(m, k)$, where $m$ is the dimension of the Krylov subspace and $k$ is the maximal dimension of the invariant subspace.

The second approach is to explicitly deflate the eigenvectors from the matrix. For example, by solving the equation

$$
\left(I-\sigma u v^{T}\right) A M^{-1} x=\left(I-\sigma u v^{T}\right) b,
$$

where $M$ is a preconditioner. Also, $v$ is a left eigenvector of $A M^{-1}$ and $u$ is arbitrary vector but is often chosen to be equal to $v$. This sort of algorithm has been proposed by Erhel et al. [10], and Burrage and Erhel [12]. Erhel et al. [10] proposed Deflated-GMres $(m, k)$ algorithm. This algorithm is updatethe preconditioner $M$ at each restart. One another algorithm is proposed by Burrage and Erhel [12] which is called $\operatorname{Deflation}(m, k)$ algorithm. This algorithm is designed a new preconditioning deflation which continuously updates the vectors in $U$, where the matrix $U$ is a basis of approximated invariant subspace. The detailed derivation of this algorithm is given in their paper [12]. 
TABLE 1: AP3000 specification

\begin{tabular}{l|l}
\hline \hline Interprocessor networks & $\begin{array}{l}\text { AP-Net 200MB/sec } \\
\text { Barrier synchronization }\end{array}$ \\
\hline Cell processor & UltraSPARC IU + FPU \\
Cache memory & $512 \mathrm{~KB}$ \\
Local memory & $256 \mathrm{MB}$ \\
\hline
\end{tabular}

\section{$3 \quad$ Numerical Experiments}

In this section we describe numerical experiments which compare parallel performance of the algorithms described in previous section on the test problem and in multiple instruction multiple data (MIMD) machine AP3000 [14]. As a preparation for the experiments, we have a brief introduction of AP3000. This machine is the workstation cluster, and each processor is made by UltraSPARC chip which runs at 300MHz. The specification of AP3000 is given in Table 1. We also use MPI as communicative library [8].

In all test runs, we utilize the components of initial guess $x_{0}=0$ and double precision arithmetic. For the sake of simplicity, we use the simple stopping criterion $\left(\left\|r_{m}\right\|_{2} /\left\|r_{0}\right\|_{2}\right) \leq 0.1 \times 10^{-12}$, where $r_{m}$ is the residual vector at $m$ th iteration.

The convergence behaviour of the deflated $\operatorname{GMRES}(m)$ algorithms is now 


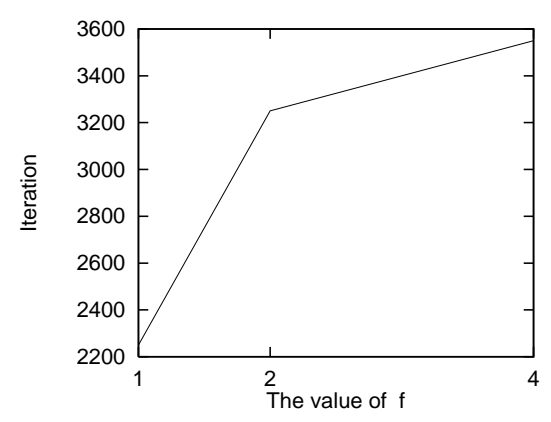

FiguRE 1: The relation between number of iterations and $f$ for DEFLATEDGMRES $(50,4)$ method in example 1.

illustrated by simple example.

\subsection{Example 1}

We now consider the following linear system of equations $A x=b$ :

$$
A=\left(\begin{array}{ccccc}
1 & 0.1 & & & 0 \\
& 2 & 0.1 & & \\
& & \ddots & \ddots & \\
& & & \ddots & 0.1 \\
0 & & & & 16384
\end{array}\right), \quad b=\left(\begin{array}{c}
1 \\
1 \\
\vdots \\
1 \\
1
\end{array}\right),
$$


where $A \in R^{16384 \times 16384}$. This example is chosen from Morgan $[4,7]$. The coefficient matrix is a triangular matrix and its eigenvalues are therefore real and are diagonal elements. You see that the distribution of eigenvalues are distinct and well separated. This problem is somewhat artificial but has been used. We solve the linear system (3) using standard $\operatorname{GMrES}(m)$, MORGan $(m, k)$, Deflated-GmRes $(m, k)$ and $\operatorname{DEFLation~}(m, k)$ algorithms. For the deflated GMRES algorithms stated above, these consist of adding approximate eigenvectors obtained from previous Arnordi step to Krylov subspace. The test uses two subspaces dimension of 50 and 70 , the last 2 and 4 of which are approximate eigenvectors. For example, these are denoted by deflated $\operatorname{Gmres}(50,2), \cdots$, deflated $\operatorname{Gmres}(70,4)$.

At first, we will decide how many eigenvalues are deflated at each restart. Therefore, we investigate the relation between iteration and the number of eigenvectors $f$ which added by each restart frequency. Figure 1 shows representative plots of above relations. From this figure, we can get the result of minimum iteration at $f=1$. So we set $f=1$.

The convergence results of numerical experiments for these algorithms are given in Table 2. Runs for which convergence was not possible in 30 minutes are labelled by $(\ldots)$. In this table, it can be seen that the convergence for $\operatorname{Morgam}(m, k)$ and DEFLATED-GMres $(M, k)$ algorithms are always better than for the standard restarted $\operatorname{GMRES}(m)$ algorithm when measured in terms of both time and iterations. On the other hand, the $\operatorname{DEFLATION}(m, k)$ algorithm could not converge at all for this problem. 
TABLE 2: The numerical result in Example 1 (time: computational time(sec), iter: the number of iterations).

\begin{tabular}{r|c|c}
\hline \hline Algorithm & time & iter \\
\hline GMRES(50) & 418 & 4100 \\
GMRES(70) & 450 & 3220 \\
\hline MORGAN $(48,2)$ & $\underline{255}$ & 1848 \\
MORGAN $(46,4)$ & 255 & 1746 \\
MORGAN $(68,2)$ & 367 & 1748 \\
MORGAN $(66,4)$ & 315 & 1396 \\
\hline DEFLATED-GMRES $(50,2)$ & 358 & 3250 \\
DEFLATED-GMRES $(50,4)$ & 261 & 2250 \\
DEFLATED-GMRES $(70,2)$ & 330 & 2240 \\
DEFLATED-GMRES $(70,4)$ & 260 & 1680 \\
\hline DEFLATION $(50,2)$ & $\ldots$ & $\ldots$ \\
DEFLATION $(50,4)$ & $\ldots$ & $\ldots$ \\
DEFLATION $(70,2)$ & $\ldots$ & $\ldots$ \\
DEFLATION $(70,4)$ & $\ldots$ & $\ldots$ \\
\hline
\end{tabular}




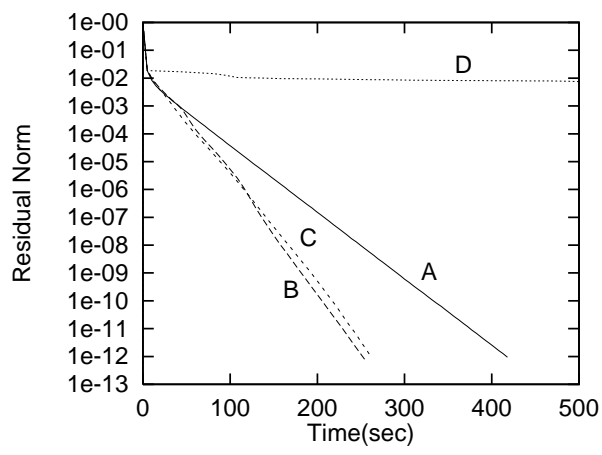

(a) Time vs. Residual norm

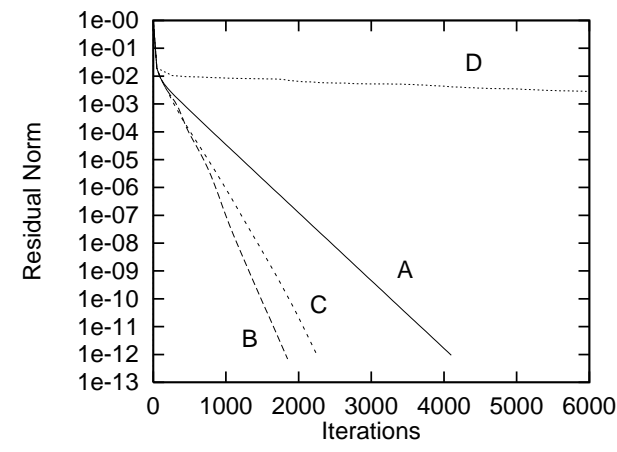

(b) Iterations vs. Residual norm

Figure 2: The convergence history of the residual norm in Example 1, A: $\operatorname{amres}(50)$, B: morgan $(48,2)$, C: Deflated-Gmres(50,4), D: Defla$\operatorname{TION}(50,4)$. 
Figure 2 shows the graph of time vs. residual and of iterations vs. residual norm, respectively. From this figure, $\operatorname{MORGAN}(m, k)$ and DEFLATED$\operatorname{GMRES}(m, k)$ algorithms have a fairly good convergence. Both algorithms have similar convergence slopes toward the final phase of the iteration. It can be seen that deflation is effective in speedup convergence early in the solution process. But, the convergence behaviour of $\operatorname{DEFLATION}(m, k)$ algorithm seems fairly flat and nearly stagnate for a large period of the convergence history.

\subsection{Example 2}

We consider the following three-dimensional problem [3]

$$
\begin{aligned}
-u_{x x}-u_{y y}-u_{z z}+R u_{x} & =g(x, y, z) \text { on } \Omega, \\
\left.u(x, y)\right|_{\partial \Omega} & =0
\end{aligned}
$$

where $\Omega \equiv[0,1] \times[0,1] \times[0,1]$, and $R$ is a Reynolds number. The right hand side is $g(x, y, z)$ is chosen so that the solution is

$$
u(x, y, z)=\exp (x y z) \sin (\pi x) \sin (\pi y) \sin (\pi z) .
$$

We discretize using central finite difference scheme on the uniform $80 \times 80 \times 80$ grid, producing a linear system of order $n=51200$.

At first, as same as the Example 1, we will decide to how many eigenvalues are deflated at each restart. We investigate the relation between iterations 


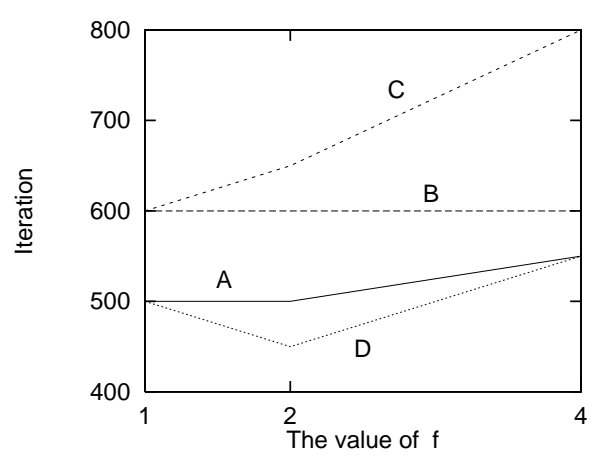

(a) DEFLATED-GMRES $(50,4)$

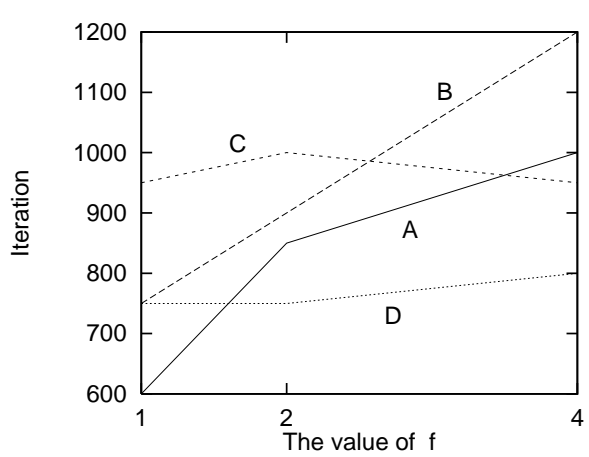

(b) DEFLATiOn $(50,4)$

Figure 3: The relation between number of iterations and $f$ for DEFLATEDGMres $(50,4)$ method and Deflation $(50,4)$ method in Example 2, A: $R=$ 1.0, B: $R=10.0, \mathrm{C}: R=100.0, \mathrm{D}: R=1000.0$.

and number of deflated eigenvalues $f$. In Figure 3, we show the relation between iterations and $f$ for Deflated-GMRES $(50,4)$ and Deflation $(50,4)$ algorithms. In this figure, we can get the minimum number of iterations, when we use $f=1$ in most cases. So we set $f=1$.

The convergence results of numerical experiments are given in Table 3 for various values of $R$. We also compare the standard $\operatorname{GMRES}(m)$ with incomplete LU (ILU) decomposition in our experiments. The ILU decomposition is frequently used for preconditioning technique, but its process includes forward and backward substitution (see Bruaset [6]). This is particularly difficult to implement on MIMD parallel computer. Bastian et al. [5] proposed 
some convenient techniques (i.e. block divided method) for parallelizing the ILU preconditioner for the linear system coming from finite difference discretization of elliptic PDE problem (see Nodera [11]). However, since the communication overhead is significantly large in some case, the ILU preconditioner can not often perform well. So, we use the modified parallelization technique of ILU decomposition which based on the original block divided method. This new algorithm decreases the communication overhead and determines the appropriate band-size. In our numerical tests, we implement the ILU decomposition using the block divided technique. The detailed implementation of ILU decomposition is given by Moriya et al. [13].

This table shows the convergence of deflated GmRes algorithms. Mostly $\operatorname{DEFLATED-GMRES}(m, k)$ algorithm is better convergence in terms of computational time. Especially, for low Reynolds numbers DefLATED-GMres $(m, k)$ algorithm converges rather fast compared to the other algorithms. However, the standard GMRES $(m)$ with ILU decomposition fairly good convergence in case of higher order Reynolds number such as $R=100$ and 1000. For DEFLA$\operatorname{TION}(m, k)$ algorithm, the eigenvalue calculation procedure takes more time than the other deflation algorithms. It is the cause that its computational time has not been reduced.

Figure 4 shows the reduction of in residual norm as deflated $\operatorname{GmRES}(m, k)$ and standard $\operatorname{GMRES}(m)$ algorithm for $R=1$. As we observed, all the curves, except the standard GMRES(50) curve, have similar convergence slopes at the early phase of iteration. The first about 100 steps of all the algorithms are identical. Differences appear at around this step 100, each algorithms 


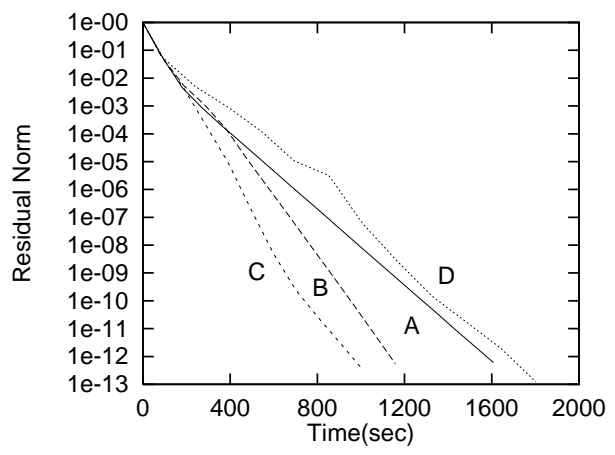

(a) Time vs. Residual norm

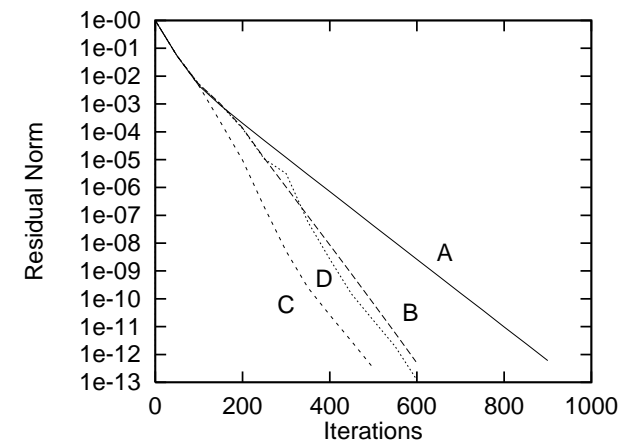

(b) Iterations vs. Residual norm

FiguRE 4: The convergence history of the residual norm in Example 2 $(R=1.0)$, A: Gmres(50), B: Morgan(48,2), C: Deflated-Gmres $(50,4)$, D: Deflation $(50,4)$. 
TABLE 3: The numerical result in Example 2 (time: computational time (second), iter: the number of iterations).

\begin{tabular}{r|c|c|c|c|c|c|c|c}
\hline \multirow{2}{*}{ Algorithm } & \multicolumn{7}{|c|}{$R$} \\
\cline { 2 - 9 } & \multicolumn{2}{|c|}{1.0} & \multicolumn{2}{c|}{10.0} & \multicolumn{2}{c|}{100.0} & \multicolumn{2}{c}{1000.0} \\
\cline { 2 - 9 } & time & iter & time & iter & time & iter & time & iter \\
\hline GMRES(50) & 1607 & 900 & 1233 & 700 & 1461 & 800 & 1183 & 650 \\
GMRES(70) & 1705 & 700 & 2046 & 840 & 2041 & 840 & 1694 & 700 \\
\hline GMRES(50)+ILU & 1515 & 450 & 1373 & 400 & $\underline{877}$ & 250 & $\underline{693}$ & 200 \\
GMRES(70)+ILU & 1658 & 350 & 1679 & 350 & 1367 & 280 & 1346 & 280 \\
\hline MORGAN(48,2) & 1158 & 598 & 1360 & 698 & 1845 & 948 & 1696 & 848 \\
MORGAN(46,4) & 1314 & 646 & 1419 & 696 & 1944 & 946 & 1871 & 896 \\
MORGAN(68,2) & 1462 & 558 & 1856 & 698 & 2199 & 838 & 2253 & 838 \\
MORGAN(66,4) & 1701 & 626 & 1923 & 696 & 2697 & 976 & 2452 & 906 \\
\hline DEFLATED-GMRES(50,2) & $\underline{963}$ & 500 & 1258 & 650 & 1568 & 800 & 1171 & 600 \\
DEFLATED-GMRES(50,4) & 1008 & 500 & $\underline{1195}$ & 600 & 1221 & 600 & 1025 & 500 \\
DEFLATED-GMRES(70,2) & 1270 & 490 & 1425 & 560 & 1970 & 770 & 1786 & 700 \\
DEFLATED-GMRES(70,4) & 1299 & 490 & 1493 & 560 & 1490 & 560 & 1503 & 560 \\
\hline DEFLATION(50,2) & 1730 & 600 & 2194 & 750 & 2557 & 850 & 2631 & 900 \\
DEFLATION(50,4) & 1802 & 600 & 2103 & 700 & 2899 & 950 & 2258 & 750 \\
DEFLATION(70,2) & 2099 & 560 & 2938 & 770 & 3163 & 840 & 2905 & 770 \\
DEFlation(70,4) & 2417 & 630 & 2687 & 700 & 3342 & 840 & 3568 & 910 \\
\hline
\end{tabular}


converge quite different ways. It is interesting to note that in this case DEFLATED-GMRES $(50,4)$ algorithm performs better convergence history than the others.

\subsection{Example 3}

We consider a model of the 3-dimensional Navier-Stokes problem stated as follows [9].

$$
a_{1} u_{x x}+a_{2} u_{y y}+a_{3} u_{z z}+R\left(a_{4} u_{x}+a_{5} u_{y}+a_{6} u_{z}\right)+a_{7} u=g(x, y, z) \quad \text { on } \Omega
$$

where

$$
\begin{aligned}
& a_{1}=2+\sin (2 \pi x) \cos (2 \pi y) \cos (2 \pi z) \\
& a_{2}=2+\cos (2 \pi x) \sin (2 \pi y) \cos (2 \pi z) \\
& a_{3}=2+\cos (2 \pi x) \cos (2 \pi y) \sin (2 \pi z) \\
& a_{4}=\sin (4 \pi x), \quad a_{5}=\sin (4 \pi y), \quad a_{6}=\sin (4 \pi z) \\
& a_{7}=\sin (2 \pi x) \sin (2 \pi y) \sin (2 \pi z)
\end{aligned}
$$

and the parameter $R$ simulates a Reynolds number. We pose equation (4) on $\Omega=[1,0] \times[1,0] \times[1,0]$ with Dirichlet boundary conditioned and discretize using central difference scheme on the uniform $64 \times 64 \times 64$ grid, producing a linear system of order of $n=262144$. The right-hand side is determined so that the exact solution is

$$
u(x, y, z)=\sin (2 \pi x) \cos (2 \pi y) \sin (2 \pi z) .
$$




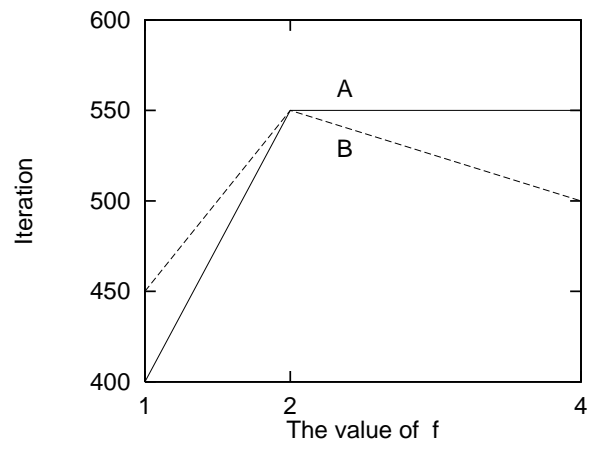

(a) DEFLATED-GMRES $(50,4)$

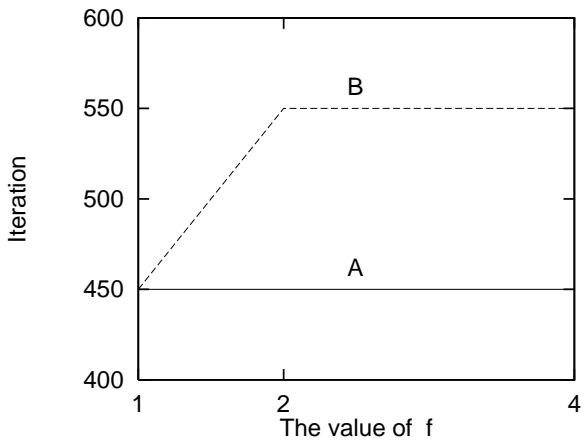

(b) DefLation $(50,4)$

Figure 5: The relation between number of iterations and $f$ for DEFLATEDGmRes $(50,4)$ method and Deflation $(50,4)$ method in Example 3, A: $R=$ 1.0, B: $R=10.0$.

Figure 5 shows that the relation between number of iterations and $f$ for Deflated-GmRes $(50,4)$ and Deflation $(50,4)$ algorithm in Example 3. From this figure, we will set $f=1$.

Table 4 shows the results of numerical experiments for various value of $R$. Run for which convergence was not possible in 70 minutes are labelled by $(\ldots)$. In this table, it can be seen that the convergence of deflated $\operatorname{GMRES}(m, k)$ algorithm is better convergence in terms of the computational time. Most significantly, the DEFLATED-GMres $(50,4)$ and DEFLATEDGMRES $(70,4)$ algorithms gave somewhat better residual reduction in some experiments. In case of $R=100$ and $R=1000$, the standard $\operatorname{GMRES}(m)$ al- 
gorithm has failed to converge, but the most of both $\operatorname{MORGAN}(m, k)$ and DEFLATED-GMRES $(m, k)$ algorithms have fairly good converged. For the Reynolds number $R=1000$, all algorithms fail to converge.

Figure 6 plotted the convergence history for these runs at $R=100$. In this figure, we can see that cases where an initial decrease in the residual norm is followed by stagnation. The first 100 steps of all the algorithms have a similar convergence slopes toward the final phase of iterations. Differences appear at around step 100. MORGAN $(48,2)$ and DEFLATED-GMRES $(50,4)$ algorithms are still identical until final convergence step. At around step 100, the standard GMRES(50) and DEFLATION $(50,4)$ algorithms are referred to as stalling. This is sometime occurs for the case of indefinite matrix. This figure also shows that the MORGAN $(48,2)$ and DEFLATED-GMRES(50,4) algorithms keep the residual size better behaved than the standard GMRES $(m)$ and DEFLATION $(m, k)$ algorithms over the course of run. Especially, MORGAN $(48,2)$ is slightly better than DEFLATED-GMRES $(50,4)$ algorithm.

\section{Concluding Remark}

We have compared in detailed the convergence behaviour of three different types of deflated GMRES algorithms for solving a linear system of equation (1) and have exposed some important features of deflated GMRES algorithms. Numerical experiments have shown that the DefLATED-GmRes $(m, k)$ algorithm is competitive with and more efficient than the standard GMRES $(m)$ 
TABLE 4: The numerical result in Example 3 (time: computational time (second), iter: the number of the iterations).

\begin{tabular}{r|c|c|c|c|c|c|c|c}
\hline \hline \multirow{2}{*}{ Algorithm } & \multicolumn{10}{|c|}{$R$} \\
\cline { 2 - 9 } & \multicolumn{2}{|c|}{1.0} & \multicolumn{2}{|c|}{10.0} & \multicolumn{2}{c|}{100.0} & \multicolumn{2}{c}{1000.0} \\
\cline { 2 - 9 } & time & iter & time & iter & \multicolumn{1}{|c|}{ time } & iter & time & iter \\
\hline GMRES(50) & 523 & 550 & 528 & 550 & $\ldots$ & $\ldots$ & $\ldots$ & $\ldots$ \\
GMRES(70) & 620 & 490 & 622 & 490 & $\ldots$ & $\ldots$ & $\ldots$ & $\ldots$ \\
\hline GMRES(50)+ILU & 717 & 400 & 700 & 400 & $\ldots$ & $\ldots$ & $\ldots$ & $\ldots$ \\
GMRES(70)+ILU & 643 & 280 & 642 & 280 & $\ldots$ & $\ldots$ & $\ldots$ & $\ldots$ \\
\hline MORGAN(48,2) & 467 & 448 & $\underline{471}$ & 448 & 633 & 598 & $\ldots$ & $\ldots$ \\
MORGAN(46,4) & 541 & 496 & 542 & 496 & 705 & 646 & $\ldots$ & $\ldots$ \\
MORGAN(68,2) & 587 & 418 & 582 & 418 & 716 & 488 & $\ldots$ & $\ldots$ \\
MORGAN(66,4) & 599 & 416 & 707 & 486 & $\ldots$ & $\ldots$ & $\ldots$ & $\ldots$ \\
\hline DEFLATED-GMRES(50,2) & 568 & 550 & 577 & 550 & $\ldots$ & $\ldots$ & $\ldots$ & $\ldots$ \\
DEFLATED-GMRES(50,4) & $\underline{428}$ & 400 & 489 & 450 & 820 & 750 & $\ldots$ & $\ldots$ \\
DEFLATED-GMRES(70,2) & 662 & 490 & 669 & 490 & 1502 & 1120 & $\ldots$ & $\ldots$ \\
DEFLATED-GMRES(70,4) & 583 & 420 & 585 & 420 & $\underline{488}$ & 350 & $\ldots$ & $\ldots$ \\
\hline DEFLATION(50,2) & 764 & 500 & 677 & 450 & $\ldots$ & $\ldots$ & $\ldots$ & $\ldots$ \\
DEFLATION(50,4) & 709 & 450 & 707 & 450 & $\ldots$ & $\ldots$ & $\ldots$ & $\ldots$ \\
DEFLATION(70,2) & 848 & 420 & 992 & 490 & 3872 & 1820 & $\ldots$ & $\ldots$ \\
DEFLATION(70,4) & 844 & 420 & 1003 & 490 & $\ldots$ & $\ldots$ & $\ldots$ & $\ldots$ \\
\hline
\end{tabular}




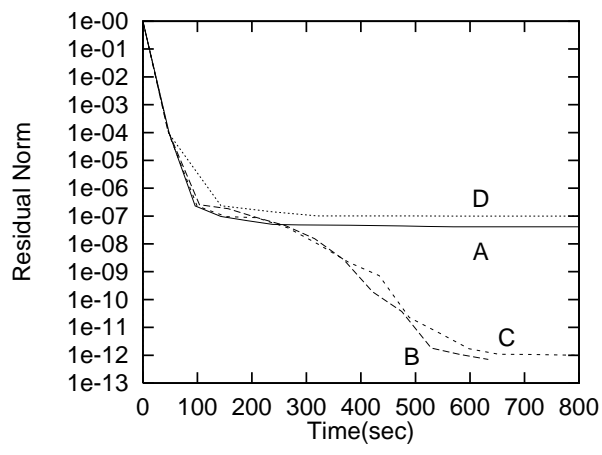

(a) Time vs. Residual norm

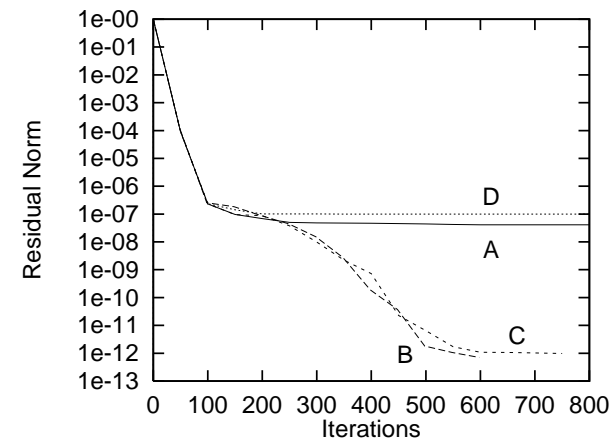

(b) Iterations vs. Residual norm

Figure 6: The convergence history of the residual norm in Example 3 ( $R=$ 100.0), A: Gmres(50), B: Morgan(48,2), C: Deflated-Gmres(50,4), D: $\operatorname{DEFLATION}(50,4)$. 
in most cases. Here we comment that it seems likely that DEFLATED$\operatorname{GMRES}(50,4)$ is better reduction of residual norm than the other algorithms.

There still remain several problems to be studied. For example, how to choose $m$ adaptively during the process of iterations, and how to choose the total number of eigenvalues are deflated, and how many eigenvalues that are deflated at each restart, when $A$ is general nonsymmetric coefficient matrix. For determining $m$ automatically when to restart, an approach of the adaptive procedure of GMRES and deflated GMRES algorithms will be given in Tsuno et al. [15] and Moriya et al. [16], respectively.

\section{References}

[1] J.H. Wilkinson. The Algebraic Eigenvalue Problem. Oxford University Press, 1965.

[2] Y. Saad and M.K. Schultz. GMRES: A generalized minimal residual algorithm for solving nonsymmetric linear systems. SIAM J. Sci. Stat. Comput., 7:856-869, 1986.

[3] W. Schönauer. Scientific Computing on Vector Computers. North Holland, 1987.

[4] R. Morgan. Computing interior eigenvalues of large matrices. Linear Algebra and its Appl., 154,156:289-309,1991. 
[5] P. Bastian and G. Horton. Parallelization of robust multigrid methods: ILU factorization and frequency decomposition Method. SIAM J. Sci. Stat. Comput., 12:1457-1470, 1991.

[6] A.M. Bruaset. A Survey of Preconditioned Iterative Methods. Pitman Res. Note in Math., No. 32, Longman, U. K., 1995.

[7] R. Morgan. A restarted GMRES method augmented with eigenvectors. SIAM J. Matrix Anal. App., 16:1154-1171, 1995.

[8] Message Passing Interface Forum, MPI: A message-passing standard, May (1995). http://www.mcs.anl.gov/mpi/

[9] G.L.G. Sleijpen and H.A. Van der Vorst. An overview of approaches for the stable computation of hybrid BiCG methods. Appl. Numer. Math., 19:235-254, 1995.

[10] J. Erhel, K. Burrage, and B. Pohl. Restarted GMRES preconditioned by deflation. Journal of Comput. and Appl. Math., 69:303-318, 1996.

[11] T. Nodera and N. Tsuno. The parallelization of the incomplete LU factorization on AP1000. Springer Lect. Notes in Comp. Sci., 1470:788-792, 1998.

[12] K. Burrage and J. Erhel. On the performance of various adaptive preconditioned GMRES strategies. Numer. Linear Algebra with Appl., 5:101-121, 1998. 
[13] K. Moriya and T. Nodera. Parallelization of ILU decomposition for elliptic boundary value problem of the PDE on AP3000. Springer, Lect. Notes on Comp. Sci., 1615:344-353, 1999.

[14] Fujitsu Lab.: Parallel server AP3000:

http://www.fujitsu.co.jp/hypertext/Products/Info_process/hpc/ap3000-e. C1000 C999 C1006 C1003 C1007 C1007 C997, C1000, C1003 C1001 C1011 C997, C1000, C1000 C1008 C997, C1000, C1000, C1000 C1008 C1001

[15] N. Tsuno and T. Nodera. The speedup of the GMRES $(m)$ method using the early restarting procedure, (in Japanese). Transaction of Information Processing Society of Japan, 40:(4)1760-1773, 1999. C1016

[16] K. Moriya and T. Nodera. The DEFLATED-GMRES $(m, k)$ method with switching the restart frequency dynamically. submitted to Numer. Linear Algebra with Appl., 1999. C1016 